\title{
Multiwavelength Erbium-doped Fiber Ring Laser with Adjustable Line Power using Variable Attenuators and DWDM
}

\author{
Hojoon Lee $^{\dagger}$ \\ Optical Communication Laboratory, Department of Information and Communication, Hoseo University, Asan 336-795, Korea
}

(Received April 2, 2014; Revised manuscript June 3, 2014; Accepted June 5, 2014)

\begin{abstract}
The homogeneous line broadening in EDF leads to unstable lasing and strong mode competition. In this paper a multiwavelength fiber ring laser that overcomes these effects by using variable attenuators between the DWDM and $1 \times \mathrm{N}$ coupler, has been proposed. Fiber ring lasers with four and eight wavelength outputs are implemented, and we show that the output powers can be adjusted with stable operation. The EDF ring laser has one four-wavelength output and four of single wavelengths, with OSNR of 50 and $60 \mathrm{~dB}$ respectively.
\end{abstract}

Keywords: Fiber laser, Ring laser, EDF laser, Multiwavelengh

OCIS codes: (140.3500) Lasers, erbium; (060.2320) Fiber optics amplifiers and oscillators; (140.3510) Laser fiber

\section{INTRODUCTION}

Multiwavelength lasers have been extensively investigated for WDM communication systems, sensors, photonics truetime-delay (TTD) of beam-forming systems and optical signal processing. ${ }^{[1]}$ Especially, the fiber ring laser using EDF (erbium-doped fiber) as a gain medium has been commonly used because of its simple configuration, high extinction ratio, cost effectiveness and low insertion loss. However, the large homogeneous line broadening in EDF leads to the unstable lasing and the strong mode competition at room temperature. As a result, the gain will be clamped by the resonator loss at only one lasing frequency. To overcome the effects, the separate gain medias have to be used for each wavelength channel. ${ }^{[2-4]}$ These fiber laser are used EDFAs same as the number of lasing lines.

Several techniques to suppress the effect of homogeneous line broadening at room temperature such as immersing in liquid nitrogen cooling EDF down to a cryogenic temperature for making it become a nonhomogeneously broadening medium, ${ }^{[5]}$ utilizing a frequency-shifted feedback technique insert an acousto-optic frequency shifter into the resonant cavity to achieve multi-wavelength oscillation at room temperature ${ }^{[6]}$ using twin-core fiber ${ }^{[7]}$ and using photonic crystal fiber. ${ }^{[8]}$ The acousto-optic frequency shifter is replaced by a sine phase modulator composed of a several-meter fiber around a cylindrical piezoelectric transducer $(\mathrm{PZT})^{[9]}$ and was complemented with a gain flattening filter for output power uniformity. ${ }^{[10]}$ Subsequently, the insertion loss is reduced and source-driven frequency is lowered. However, the output power uniformity is very poor owing to no effort made to equalize the output powers of lasing lines. The uniformity is further improved by Slavik et al., ${ }^{[11]}$ but the cavity loss is high, which increases the amplified spontaneous emission (ASE) noise at lasing wavelengths. Many of fiber lasers do not have uniform output power at each lasing lines.

In this paper, we have proposed and experimentally demonstrated a multiwavelength EDF ring laser using variable attenuators, which have adjustable output powers of lasing lines at each wavelength. The fiber laser has an output port of multiwavelength and output ports for each single wavelength.

\section{PRINCIPLES OF OPERATION AND SYSTEM CONFIGURATION}

Figure 1 shows a configuration of multiwavelength EDF ring laser which is capable of adjusting the power of each lasing lines. In the multiwavelength EDF ring laser, a Fabry-Perot filter is used to select for the lasing line wavelengths. Polarization controller (PC), isolators and optical coupler are used. In this paper, we used DWDM (dense-wavelength division multiplexing) to divide the lasing lines and $1 \times \mathrm{N}$ coupler to combine wavelengths, and attenuators to adjust the attenuations of each lasing lines suppressing the homogeneous line broadening of

\footnotetext{
${ }^{\dagger}$ E-mail: hojoon@hoseo.edu

Color versions of one or more of the figures in this paper are available online.
} 
EDF at room temperature.

Initially, ASE of EDF pass through the FP (Fabry-Perot) filter and the wavelengths of each lasing lines are divided into each channel bandwidths of DWDM. The channel separation of the DWDM and FSR (free spectral range) of FP filter should be almost the same.

The power of lasing lines are divided by four of $1 \times 2$ couplers (4 of single wavelength outputs), therefore one half of the line power emitted to single wavelength laser output and the rest of the line power launched into attenuator which make a equal gain of the ring for the uniform lines power. $1 \times 4$ coupler is used to add powers of separated channels and can be replaced by other DWDM to reduce the coupling loss of the ring laser. DWDM, variable attenuators and $1 \times 4$ optical coupler can adjust resonator loss for each lasing lines to overcome the mode competition in lasers with homogeneous line broadening, The 30:70 of output coupler (multiwavelength output) is used as multiwavelength output laser output port and the polarization controller is used.

\section{EXPERIMENTAL RESULTS OF OPTICAL FIBER RING LASER}

Figure 2 shows the output spectrum of the fiber ring laser, when the variable attenuators are removed in the multiwavelength fiber laser configuration of Fig. 1. OSA (optical spectrum analyzer), Anritsu MS9710B is used to measure the optical spectrum. We can observe unstable oscillation and mode competition that result from the homogeneous line broadening in $\mathrm{EDF}$ at the room temperature.

Figure 3 shows the output spectrum of proposed multiwavelength fiber ring laser after the variable attenuators are connected as shown in Fig. 1 and adjusted. The multiwavelength EDF ring laser is operate well overcoming the homogeneous line

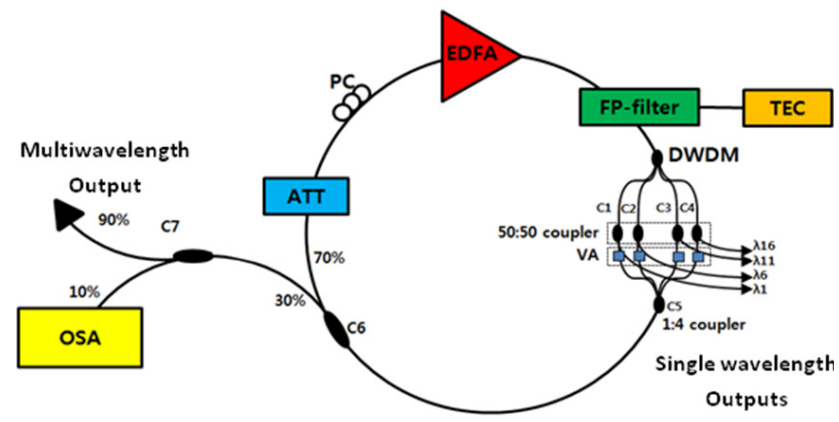

FIG. 1. The proposed configuration of variable output channel power erbium-doped fiber ring laser.

EDFA: erbium-doped fiber amplifier; FP: Fabry-Perot filter; DWDM: dense-wavelength division multiplexing; PC: polarization controller and C1-C7: fiber couplers broadening.

The transmittance of FP filter is shown in Fig. 4, which is measured by optical dispersion analyzer (Agilent 86038A). FSR of FP filter is $\sim 1.58 \mathrm{~nm}$ in this study. 16 channels DWDM that have the channel separation of $200 \mathrm{GHz}$, the bandwidths at $-0.5 \mathrm{~dB}$ of $0.88-0.97 \mathrm{~nm}$ for the each channels and the optical

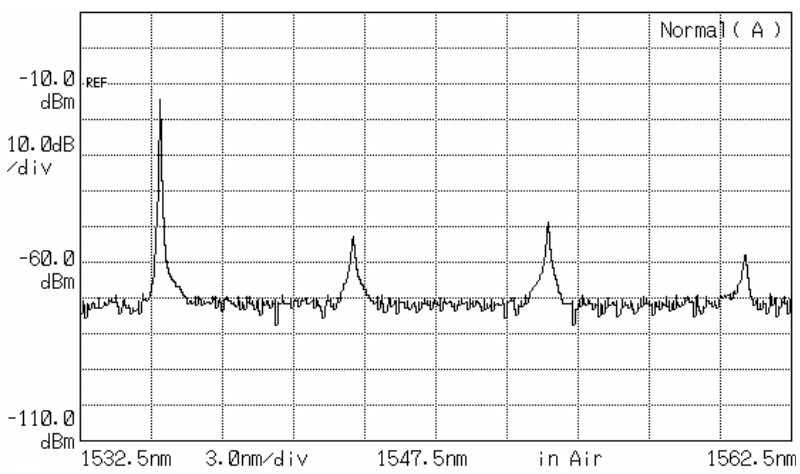

FIG. 2. Unstable output spectrum of multiwavelength EDFA based fiber laser.

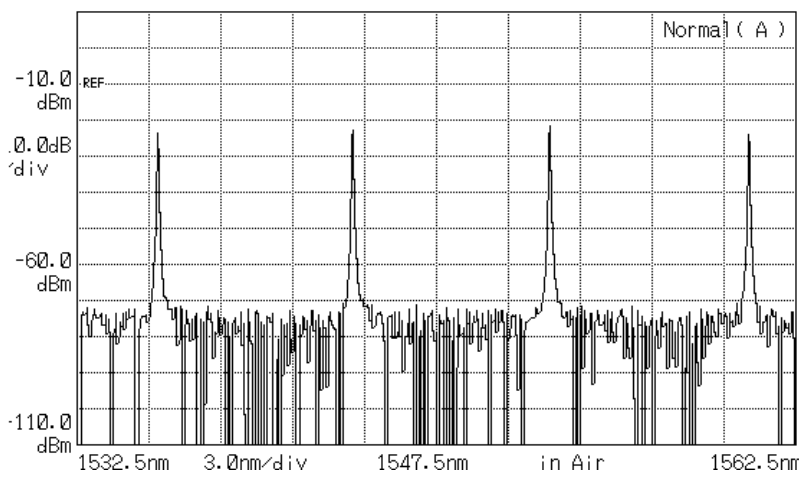

FIG. 3. Output spectrum of proposed EDF ring laser with uniform output power.

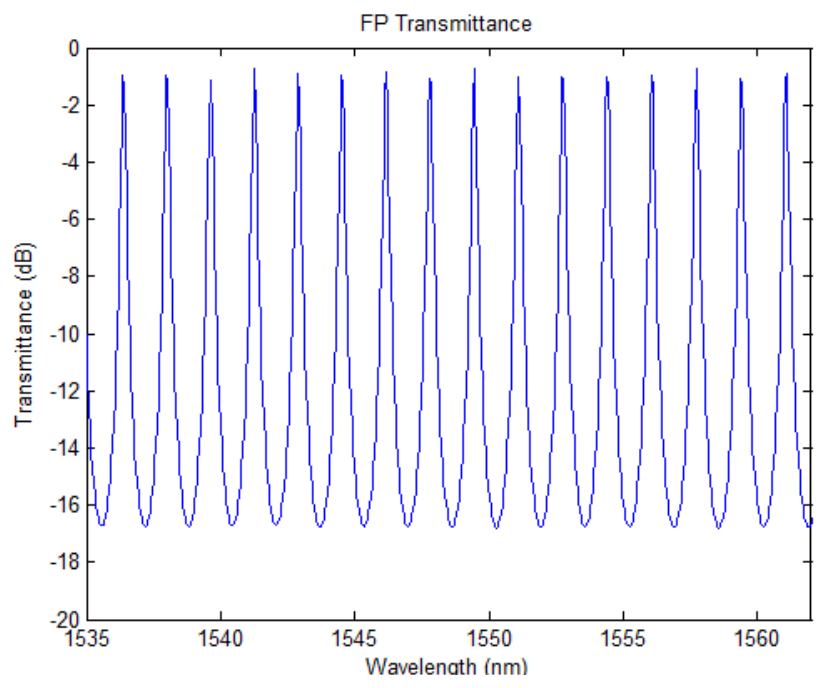

FIG. 4. Transmittance of Fabry-Perot filter. 


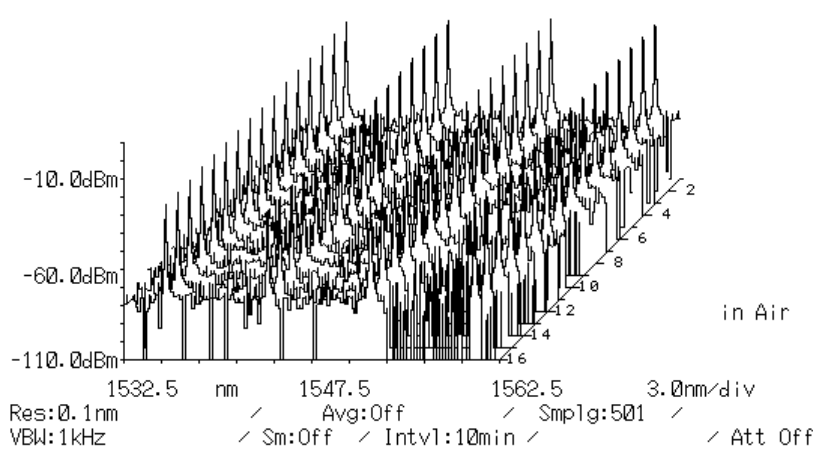

FIG. 5. Output spectrum of EDF ring laser measured 16 times with 10 minutes interval at room temperature.

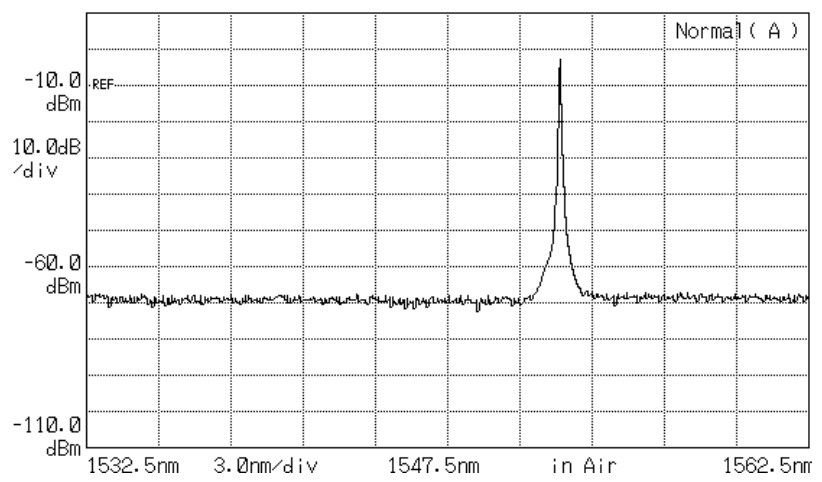

FIG. 6. Spectrum of single-wavelength output of proposed EDF ring laser at center wavelength of $1552.1 \mathrm{~nm}$.

return loss of $>45$ is used. In this experiment, because the channel 1, 6, 11 and 16 of DWDM are used, the lasing line separation is $\sim 8.2 \mathrm{~nm}$ ( 5 times of $200 \mathrm{GHz}$ ). OSNRs (optical signal to noise ratio) more than $50 \mathrm{~dB}$ for the four wavelengths of 1535.8, 1543.9, 1552.1 and $1560.5 \mathrm{~nm}$, are obtained after adjusting the attenuation of variable attenuator for gain equalization of multiwavelength line output. The power of lasing lines are about $-23 \mathrm{dBm}$ as shown in Fig. 5, which is measured on power scale of $10 \mathrm{~dB} / \mathrm{div}$ using MS9710B of Anritsu Corp.

For stability measurement of the output power of the proposed fiber ring laser, the optical spectrum analyzer is also used. Figure 5 shows the output spectra of the multiwavelength EDF laser repeatedly measured 16 times with 10 minutes interval on room temperature and the power fluctuation was measured to be less than $1 \mathrm{~dB}$. The output spectrum of the proposed multiwavelength erbium-doped fiber ring laser was stable.

Figure 6 shows spectrum for the single-wavelength output at $1552.1 \mathrm{~nm}$, which is emitted from 50:50 coupler between DWDM and $1 \times 4$ coupler. OSNR of $65 \mathrm{~dB}$ and the line powers of $-2.6 \mathrm{dBm}$ are obtained.

The 4-wavelength EDF laser with uniform power output was introduced. To increase the number of wavelengths to $\mathrm{N}$, number of DWDM channels, variable attenuators, $1 \times 2$ couplers and

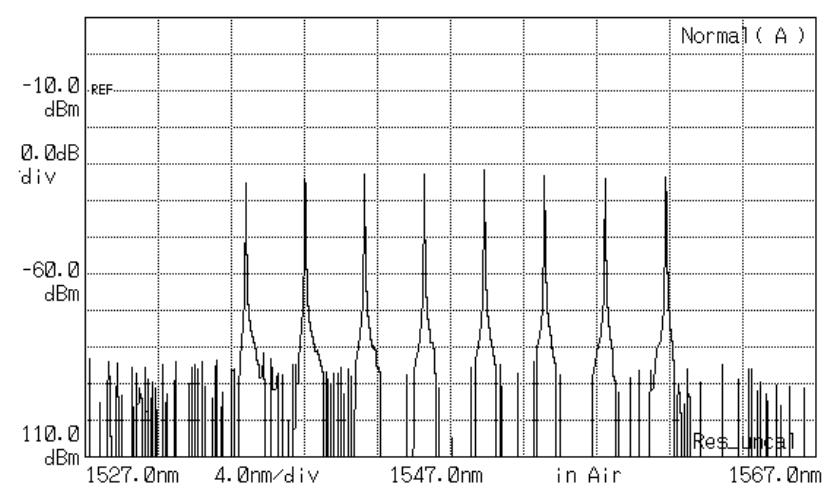

FIG. 7. Spectrum of multiwavelength outputs of proposed EDF ring laser for the case of uniform 8-line power.

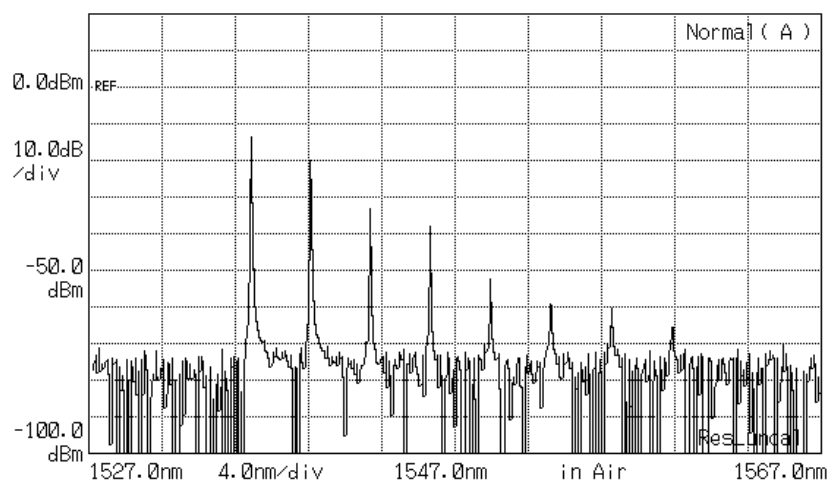

FIG. 8. Multiwavelength output spectrum of proposed EDF ring laser for the case of increasing line power as decreasing wavelength.

branch of $1 \times \mathrm{N}$ coupler should be N. Figure 7 shows the spectra of 8-wavelength outputs of proposed EDF ring laser in the case of uniform output power. The peak power of each lasing lines are about $-32 \mathrm{dBm}$ at the multiwavelength output. Figure 8 shows the spectrum of increasing output power as decreasing wavelength, when single wavelength outputs in Fig. 1 are removed. The powers for each line are between -14 and -65 $\mathrm{dBm}$. Figure 9 and 10 shows the spectrum of the increasing output power as going to center wavelength and at the odd line wavelength, respectively. These figures are show that the power distribution for lasing lines can be change adjusting variable attenuators.

In this study, the output power of lasing lines can be changed by adjusting the attenuation of variable attenuator in order to suppress homogeneous line broadening in EDF. The fiber ring laser was also implemented to obtain a multiwavelength output by using 30:70 coupler and single-wavelength outputs by using 50:50 couplers between DWDM and $1 \times \mathrm{N}$ coupler. Lasing wavelengths of proposed optical fiber ring laser could be changed in the range of DWDM bandwidth by adjusting the voltage of Fabry-Perot filter. 


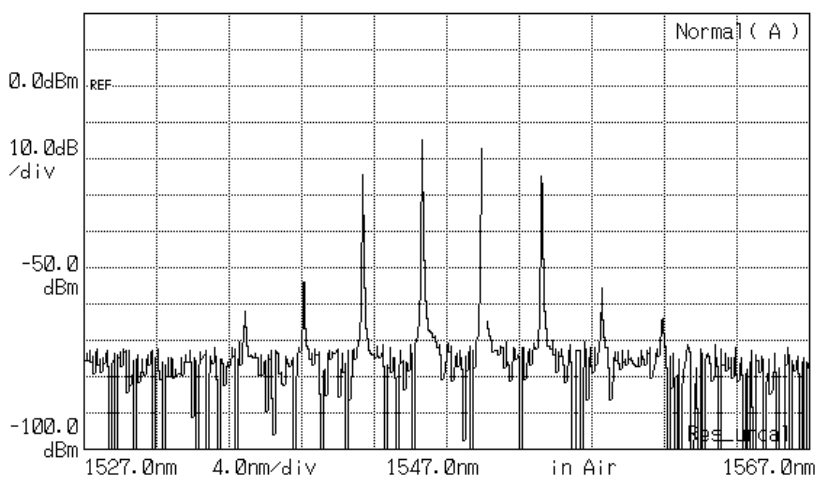

FIG. 9. Multiwavelength output spectrum of proposed EDF ring laser for the case of increasing line power as going to center wavelength.

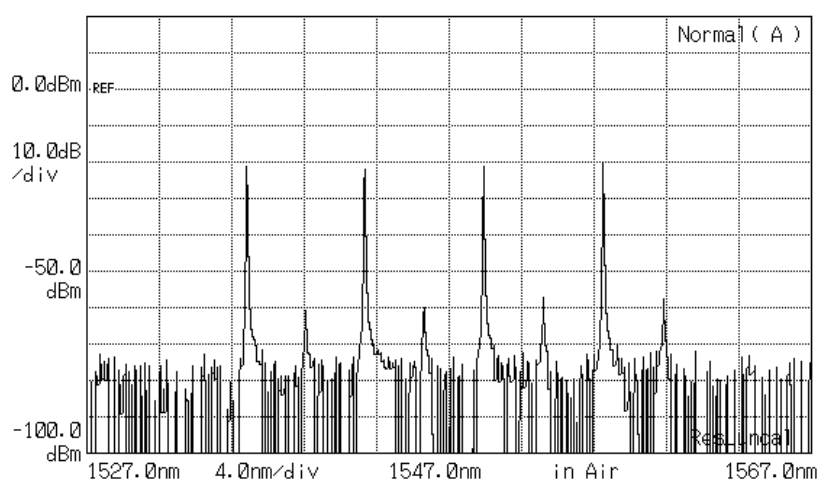

FIG. 10. Multiwavelength output spectrum of proposed EDF ring laser for the case of increasing power at the odd line wavelength.

\section{CONCLUSION}

In this paper, we proposed a multiwavelength EDF ring laser. The fiber ring laser have a multiwavelength output and $\mathrm{N}$-single wavelength outputs. The variable attenuators between the DWDM and $1 \times \mathrm{N}$ coupler in the fiber ring are used to give different gain for each lasing line wavelengths. We found that the use of variable attenuators make it possible to overcome the homogeneous line broadening of EDF on room temperature in the fiber ring laser.

The 4 and 8 -wavelength fiber ring lasers are implemented. We showed that the each output lasing line powers could adjust stably using variable attenuators. In this experiment, OSNR of implemented laser for multiwavelength and singlewavelength outputs was $\sim 50 \mathrm{~dB}$ and $\sim 65 \mathrm{~dB}$ OSNR, respectively. The fiber ring laser can be used for optical source, optical sensing, optical repeater, sensor system and photonics true-time delay for beam-forming.

\section{REFERENCES}

1. J. Chow, G. Town, B. Eggleton, M. Ibsen, K. Sugden, and I. Bennion, "Multiwavelength generation in an erbium-doped fiber laser using in-fiber comb filters," IEEE Photon. Technol. Lett. 8, 60-62 (1996).

2. H. Takahashi, H. Toba, and Y. Inoue, "Multiwavelength ring laser compound of EDFA's and an arrayed-waveguide wavelength multiplexer," Electron. Lett. 30, 44-45 (1994).

3. K.-H. Kim, H.-K. Lee, S.-Y. Park, and E.-H. Lee, "Wavelengthvarying multi-wavelength optical filter laser using a single pump light source," U.S. Patent 5524118 (1996).

4. T. Miyazaki, N. Edagawa, S. Yamamoto, and S. Akiba, "A multiwavelength fiber ring-laser employing a pair of silicabased arrayed-waveguide-gratings," IEEE Photon. Technol. Lett. 9, 910-912 (1997).

5. S. Yamashita and K. Hotate, "Multiwavelength erbium-doped fiber laser using intracavity etalon and cooled by liquid nitrogen," Electron. Lett. 32, 1298-1299 (1996).

6. A. Bellemare, M. Karasek, M. Rochette, S. LaRochelle, and M. Tetu, "Room temperature multifrequency erbium-doped fiber lasers anchored on the ITU frequency grid," J. Lightwave Technol. 18, 825-831 (2000).

7. O. Graydon, W. H. Loh, R. I. Laming, and L. Dong, "Triple-frequency operation of an Er-doped twincore fiber loop laser," IEEE Photon. Technol. Lett. 8, $63-65$ (1996).

8. A. Zhang, H. Liu, M. S. Demokan, and H. Y. Tam, "Stable and broad bandwidth multiwavelength fiber ring laser incorporating a highly nonlinear photonic crystal fiber," IEEE Photon. Technol. Lett. 17, 2535-2537 (2005).

9. K. Zhou, D. Zhou, F. Dong, and N. Q. Ngo, "Room-temperature multiwavelength erbium-doped fiber ring laser employing sinusoidal phase-modulation feedback," Opt. Lett. 28, 893895 (2003).

10. S. K. Kim, M. J. Chu, and J. H. Lee, "Wideband multiwavelength erbium-doped fiber ring laser with frequency shifted feedback," Opt. Commun. 190, 291-302 (2001).

11. R. Slavik, S. LaRochelle, and M. Karasek, "High-performance adjustable room temperature multiwavelength erbium-doped fiber ring laser in the C-band," Opt. Commun. 206, 365-371 (2002).

12. Y. G. Han, J. H. Lee, S. B. Lee, L. Poti, and A. Bogoni, "Novel multiwavelength erbium-doped fiber and raman fiber ring lasers with continuous wavelength spacing tunability at room temperature," J. Lightwave Technol. 25, 2219-2225 (2007). 